\title{
NUEVOS ESPACIOS TERCIARIOS DE BARCELONA: ADAPTACIONES A UNA ECONOMÍA GLOBALIZADA
}

\author{
POR \\ ROSA TELLO ROBIRA \\ SERGI MARTÍNEZ RIGOL \\ ASSUMPTA BOBA CABALLÉ
}

\section{Proceso de terciarización}

En el último cuarto de siglo Barcelona se ha ido conformando como un gran centro terciario. Numerosos indicadores manifiestan que la ciudad ya no es un espacio adecuado prioritariamente para la producción industrial, sino un espacio urbano que se está adaptando a los nuevos requerimientos del sistema económico globalizado; que se va modificando para dar cabida a la progresiva e intensa implantación de actividades de gestión, administración - pública y privada-, de nuevas actividades de consumo de bienes materiales e inmateriales, de cultura, ocio...

Desde mediados de los años setenta la dinámica demográfica del municipio de Barcelona se desacelera hasta ser negativa: entre 1981 y 1996 se pierden 243.822 habitantes ${ }^{1}$. El conjunto de la población barcelonesa se envejece considerablemente: el porcentaje de población de más de 65

${ }^{1}$ Según el Institut Municipal d'Estadística la población del municipio de Barcelona era de 1.754.713 habitantes en el 1975; de 1.752.627 habitantes en 1981; 1.643 .542 habitantes 1991 y de 1.508.805 habitantes según el padrón de 1996.

Rosa Tello Robira, Sergi Martínez Rigol y Assumpta Boba Caballé: Departamento de Geografía Humana, Universitat de Barcelona.

Estudios Geográficos

Tomo LXI, 2000, n. ${ }^{\circ} 238$, enero-marzo 
años era del 13,3\% en1981, en 1991 era del 17,4\% y en 1996 la proporción era de un $20,7 \%{ }^{2}$.

Muchos jóvenes han de «emigrar» de Barcelona, han de establecer su residencia fuera del municipio, incluso más allá del entorno metropolitano, a causa del excesivo precio de la vivienda respecto a sus posibilidades de financiación.

En la ciudad de Barcelona, el precio medio por $\mathrm{m}^{2}$ de vivienda de nueva construcción era de 102.712 pesetas en 1987; en 1990, 203.274 pesetas; en 1993, 234.238 pesetas y en 1998 era de 250.039 pesetas $^{3}$. Es decir que una vivienda de tamaño medio de unos $70 \mathrm{~m}^{2}$ valía aproximadamente 8.200 .000 ptas. en $1987 ; 14.300 .000$ ptas. en $1990 ; 16.400 .000$ ptas. en 1993. El precio de la vivienda aumentó en un $97,9 \%$ hasta 1990 , o en un $128 \%$ hasta 1993. Este incremento no se correspondía con el del poder adquisitivo (alrededor de un 5\% anual equivalente al índice de inflación) o con el incremento de los salarios, particularmente los de la población joven. Teniendo en cuenta que los intereses hipotecarios estaban situados, hasta el año 1994, entre el $12 \%$ y el 15\%, en 1987 se debían destinar un mínimo de 80.000 ptas. mensuales para la adquisición financiada de vivienda; en 1990 se habían de destinar unas 140.000 y en 1993 alrededor de 160.000 ptas. mensuales. A partir de 1996, el descenso progresivo del interés de los préstamos hipotecarios hasta alcanzar valores situados en torno al 4,5\%, a finales de 1999, modificó sensiblemente las condiciones de acceso a la vivienda, a pesar del aumento sostenido de los precios. La mensualidad destinada a la adquisición de vivienda se situaba alrededor de 166.000 ptas. en 1998. Teniendo en cuenta que en Barcelona la dimensión media de la vivienda de nueva construcción ha aumentado hasta $\operatorname{los} 113 \mathrm{~m}^{2}$ y que el pago de la entrada asciende a los tres o cuatro millones, la cantidad mensual destinada a la adquisición de la vivienda es muy semejante a la de 1993, o incluso inferior en valor real, ya que estos precios están cifrados en pesetas corrientes ${ }^{4}$.

Independientemente de las condiciones coyunturales más o menos favorables para el acceso a la vivienda, la capacidad de endeudamiento de gran parte de los jóvenes barceloneses siguen estando sometida a la in-

\footnotetext{
${ }^{2}$ Datos del Institut Municipal d'Estadística.

3 Valores en pesetas corrientes.

${ }^{4}$ Datos extraídos de Barcelona Economía, tercer trimestre de 1998, Ajuntament de Barcelona
} 
seguridad de los lugares de trabajo y a las condiciones de precariedad del empleo. Residir en Barcelona supone para una pareja joven disponer, por lo menos, de un sueldo fijo de unas 170.000 pesetas mensuales durante quince o veinte años, mientras que en municipios situados en un radio de $50,60 \mathrm{~km}$, o más, requiere actualmente sólo unas setenta u ochenta mil pesetas. Mientras la población de Barcelona decrece, y la ciudad se terciariza, se industrializan y aumentan demográficamente los municipios de más de cinco mil habitantes, extendiéndose progresivamente la urbanización sobre el territorio catalán ${ }^{5}$.

Desde 1970 en la ciudad de Barcelona se fueron perdiendo progresivamente lugares de trabajo del sector industrial. Esta pérdida se debió, tanto al traslado de centros de fabricación hacia la región metropolitana, como a la reestructuración económica de los diversos sectores productivos. Así, entre 1970 y 1986 se perdieron 249.861 empleos industriales y sólo se crearon 16.532 puestos de trabajo en el sector terciario. En cambio entre 1986 y 1996 se perdieron 92.336 empleos del sector industrial y se crearon 241.371 en el de servicios ${ }^{6}$. Sin embargo, hay que tener en cuenta que el empleo terciario se encuentra fuertemente polarizado entre los puestos de trabajo altamente cualificados, bien retribuidos, de los sectores punta y los empleos temporales, poco cualificados, precarios y poco retribuidos, abundantes en todos los subsectores terciarios. Los tres millones, in crescendo, de visitantes anuales que recibe Barcelona desde 1992 son un buen indicador de la transformación del sector terciario, a la vez que indicador del empleo precario y temporal, porque precisamente el sector de hostelería y restauración es uno de los que registra mayor fluctuación de los lugares de trabajo.

El proceso de terciarización incluye también un proceso de homogeneización social que está íntimamente vinculado con la transformación del espacio urbano. La renta familiar disponible media de Barcelona era de 5,5 millones de pesetas en 1998. Desde 1990 ha aumentado un 62,92 \%; en este año se situaba alrededor de los 3,4 millones de pesetas ${ }^{7}$. Si actualmente la mayoría de la población disfruta de unas rentas situadas en la franjas altas de las bases impositivas, puede decirse que la baja demográfica de

\footnotetext{
${ }^{5}$ Tello Robira, R., y Martínez Rigol, S.: «Terciarización y encarecimiento de la vivienda en Barcelona», Revista de Geografía vol. XXIX, nº 2. Jul-ept. 1995, pp41-51.

${ }^{6}$ Ajuntament de Barcelona: Barcelona Economía, diversos números

${ }^{7}$ Dato extraído de Barcelona Economía, tercer trimestre de 1998, Ajuntament de Barcelona
} 
la ciudad se ha debido en gran parte a un goteo emigratorio de la población con rentas más bajas ${ }^{8}$.

\section{Transformación del espacio urbano}

Las transformaciones de la estructura productiva y social de la ciudad de Barcelona se reflejan en el proceso de cambio de su espacio interno. Este proceso no es espontáneo ni estrictamente casual. Se trata de un proceso impulsado y dirigido por la política urbana municipal que, desde 1982, lleva a cabo una hábil estrategia para reconvertir la ciudad en un espacio adecuado para el desarrollo de los servicios y del consumo.

Concepción darwinista de la ciudad. A partir de la década de los noventa, desde las instancias públicas locales se manifiesta de nuevo un discurso cientifista sobre la ciudad concebido a través de una renovada óptica evolucionista-darwiniana. La ciudad se concibe como un elemento vivo sometido a distintos ciclos vitales dependientes de factores internos y externos: "Como elemento vivo una ciudad está sometida al ciclo de vida ligado a diversos factores: ubicación en el territorio, actividades que se desarrollan... La duración del ciclo es variable, dependiendo de diversos factores, unos ligados a los cambios del modo de producción y otros particulares ligados a la evolución de cada formación económicosocial... y a los factores del entorno» ${ }^{9}$.

Esta analogía cientifista permite a los poderes locales construir un discurso, convincente y efectivo para la mayoría de ciudadanos, sobre las actuaciones públicas en este particular «elemento orgánico». En esta perspectiva darwiniana, la «supervivencia» de dicho organismo necesariamente dependerá de la capacidad de desarrollar estrategias de adaptación a las nuevas condiciones el medio en que «viven» las ciudades, el cual sin duda se identifica con el sistema económico. Si esto es así, esta concepción requiere actualmente una neta percepción de la relación entre el lugar, es decir la ciudad, y el sistema económico global; re-

8 Tello Robira, R. y Martínez Rigol, S.: «Terciarización y èncarecimiento de la vivienda en Barcelona», Revista de Geografía, vol. XXIX, n. ${ }^{\circ}$ 2, jul-ept. 1995, pp. 41-51.

${ }^{9}$ Forn, M. de (1989), «Evolución de la programación de las administraciones públicas. Los planes estratégicos», CEUMT, n. ${ }^{\circ} 108$, pp. 29-35, Barcelona 
quiere una clara percepción de las relaciones entre el proceso de transformación urbana - «factores internos», que se pueden identificar como el proceso de terciarización anteriormente descrito- y el proceso globalización - «condicionantes externos o de entorno».

El sistema económico globalizado reposa sobre bruscas diferencias de potencial entre los espacios de producción de servicios y captación de consumo y los espacios de producción de bienes, aunque los costes de producción más bajos no permanezcan localizados siempre en el mismo $\operatorname{lugar}^{10}$. Dicho diferencial se mantendrá, si los primeros espacios mantienen las condiciones adecuadas para la captación y emisión de flujos (técnicos, capital, información, bienes, consumo); si siguen manteniendo las condiciones espaciales de determinados lugares - algunas ciudades o algunos lugares de algunas ciudades-para gestionar y hacer funcionar el propio sistema globalizado. Por tanto sus lugares concretos, especialmente las ciudades terciarizadas (lugares centrales de este sistema urbano mundializado), deberán continuamente transformar sus espacios y adaptar su morfología a las variabilidades del sistema económico globalizado.

En esta consideración darwiniana de la ciudad, el proceso de globalización se toma como el condicionante externo de las transformaciones urbanas. Si desde las mencionadas instancias públicas se identifican «los cambios del modo de producción» con el proceso de globalización (y presumiblemente es así), se justifican las políticas urbanas encaminadas a adaptar el lugar y sus condiciones, la ciudad y su espacio socio-urbano, a los imperativos del proceso de globalización.

Política urbana. La mencionada concepción organicista de la ciudad presupone necesariamente una política urbana basada en un esquema darwiniano de adaptación, supervivencia, competencia y evolución. Supone, por tanto, una política urbana fundamentada en la ciudad como un todo, como un sólo objeto viviente con capacidad de mutación, más que fundamentada en la multiplicidad de sujetos, los ciudadanos, y la diversidad de problemáticas. La subordinación de la política urbana al imperativo del

${ }^{10}$ Lantz, P. (1996), «Centralization symbolique, dispersion productive», Réseaux productifs et territoires urbains, Villes et Territoires, n. ${ }^{\circ}$ monográfico, abril, Presses Universitaires de Mirail, Toulouse, pp. 139-147. La reciente crisis del sudeste asiático es un ejemplo paradigmático de la movilidad geogáfica de la producción característica de la economía globalizada 
sistema económico global implica orientar las transformaciones del espacio urbano hacia una mayor consecución de valor de cambio, no sólo de los espacios transformados, sino del conjunto de la ciudad.

La política urbana que se ha llevado a cabo en Barcelona desde mediados de los años ochenta es compleja, se descompone en diversos frentes de actuación y está encaminada decididamente a transformar la ciudad en un espacio adaptado a la nuevas condiciones del sistema económico global.

La política urbana barcelonesa es semejante a la política empresarial: «Del mismo modo como las empresas diseñan su estrategia para hacer frente a los posibles cambios de precios, de costumbres de los consumidores o de potenciales innovaciones tecnológicas, la administración local diseña su política urbana. No existe ninguna razón para que los municipios no planifiquen también la estrategia y estudien con anticipación y detalle las actuaciones a emprender» ${ }^{11}$.

La ciudad se administra y gestiona como una empresa cuya particularidad consiste en que constantemente se está produciendo un producto final, espacio urbano, que se consume y se produce a la vez. Por una parte, se optimizan sus recursos - espacio construido, emplazamiento, comunicaciones, patrimonio histórico y cultural- para obtener un producto final -rehabilitación y mejora del espacio construido, monumentalidad del espacio público, multifuncionalidad espacial, diversidad de espacios centrales. Por otra parte, se establecen estrategias de promoción de su producto, el marketing de la ciudad.

Esta política se fundamenta en tres ejes de actuación: sobre la morfología del espacio urbano, sobre el funcionamiento económico social de la ciudad y sobre la imagen de la ciudad.

La actuación sobre la morfología del espacio urbano se emprende desde el Plan de Áreas de Nueva Centralidad, una renovada gestión de los PERI definidos y aprobados desde de inicios de los ochenta. La actuación sobre el funcionamiento socioeconómico de la ciudad se emprende desde el Plan estratégico y social. Barcelona 2000, en sus versiones $I, I I$ y $I I I^{12}$. Y la actuación sobre la imagen comprende desde el

\footnotetext{
11 Serra Ramoneda, A.: «Estatègia és previsió», Barcelona 2000, nov., 1988, p. 2.

12 Tello Robira, R: «Les stratégies de Barcelone pour l'ann 2000», Barcelone-Toulouse Horizon 2000, Villes et territoires, n. ${ }^{\circ}$ 4, 199, pp. 43-56 Presse Universitaire Toulouse-Le-
} Mirail. 
proceso de su producción hasta sus estrategias difusión ${ }^{13}$. Estas actuaciones y sus correspondientes planes se inician y se coordinan entre 1987-1988, poco después de la nominación de Barcelona como sede olímpica para 1992.

Estrategias de producción de nuevo espacio urbano. El espacio central de Barcelona no es un típico Central Business District. Las funciones centrales no han conseguido desplazar totalmente la función residencial ni las de servicios asociados a ella. Por la noche no llega a ser un desierto, además de sus habitantes, le dan vida actividades lúdico-recreativas. Durante el día, las actividades comerciales predominan por encima de cualquier otra actividad económica. Barcelona, por tanto no tiene un espacio específicamente destinado para empresas de servicios a la producción y al consumo, un espacio exclusivamente de negocios, sino que su espacio central es multifuncional, con neto predominio comercial y se extiende a lo largo de $5 \mathrm{~km}^{14}$. Nunca se desarrolló ningún tipo de plan urbanístico que se propusiera refuncionalizar el centro urbano para convertirlo en un típico CBD, tal como muchas ciudades lo hicieron en el momento que las prácticas urbanísticas se basaban en la especialización funcional del espacio, en el zoning.

Con la sucesiva puesta en práctica del Plan de Áreas de Nueva Centralidad (ANC) se pretende crear espacios especializados en funciones centrales, en muchos casos, pequeñas zonas de CBD que sustituyen la falta de un distrito central de negocios, de modo que toda la ciudad se convierta en el gran CBD metropolitano. La finalidad del Plan, según queda explícito en el texto de divulgación ${ }^{15}$, es reequilibrar las actividades terciarias del conjunto de la ciudad, de forma que con ello se llegue a romper la inercia de su localización en la zona central-comercial. Así pues, las Areas de Nueva Centralidad pueden considerarse como los nuevos polos económicos, los polos terciarios de la ciudad.

Se espera que estos espacios reconvertidos en áreas centrales actúen como elementos catalizadores de la renovación urbana de su entorno in-

Tello Robira, R: «Plano estratégico de Barcelona. Do balaço ao Novo Plano», Finisterra, vol.XXIX, n. ${ }^{\circ}$ 57, pp. 157-164, 1994.

${ }^{13}$ Benach, N.: Ciutat i producció d'imatge: Barcelona1979-1992, Tesis Doctoral, Universitat de Barcelona, 1997.

${ }^{14}$ Carreras, C.; Domingo, J., y Sauer, C.: Les àrees de concentració comercial de Barcelona, Camara de Comercio de Barcelona, 1990.

${ }^{15}$ Ajuntament de Barcelona: Pla d'Arees de Nova Centralitat, Barcelona, 1987.

$$
-151-
$$


mediato. Cada uno debe actuar de foco de atracción de que induzca a la revalorización económica y social del barrio donde está emplazada, por consiguiente, su distribución ha de influir en la aceleración del proceso de transformación urbana del conjunto de la ciudad. Asimismo las características morfológicas de cada nuevo espacio debe proporcionarle potencial de atracción y renovación.

Localización estratégica de nuevos espacios urbanos. La superficie afectada por el Plan de Áreas de Nueva Centralidad asciende a casi un millar de hectáreas $(969,61)^{16}$ que representan aproximadamente un $10 \%$ de la superficie municipal (9.800 ha). (cuadro I)

Aunque el emplazamiento de cada una de las ANC tiene que ver con la aleatoriedad de los procesos históricos que determinaron la conformación del espacio urbano de la ciudad de Barcelona, actualmente, su particular dispersión dentro del tejido urbano municipal, se considera estratégica para la transformación global de la ciudad. Unas están emplazadas en antiguos barrios industriales, otras en espacios ocupados por

CUADro I

ÁREAS DE NUEVA CENTRALIDAD

\begin{tabular}{lc}
\hline & Hectáreas \\
\hline Montjuïc & 399,40 \\
Vila Olímpica & 129,97 \\
Montbau-Val d'Hebron & 113,24 \\
Torre Melina & 40,50 \\
Diagonal-Sarrià & 34,00 \\
Calle Tarragona & 12,50 \\
Renfe-Meridiana & 35,00 \\
Plaça Glòries & 67,00 \\
Sagrera & 80,00 \\
Diagonal-Prim & 35,00 \\
& 969,61
\end{tabular}

Fuente: Memòria d'Urbanisme 1983-1987, Ajuntament de Barcelona, p. 16.

${ }^{16}$ Memòria d'Urbanisme 1983-1987, Ajuntament de Barcelona, p. 16. 
infraestructuras obsoletas, otras en espacios poco consolidados urbanísticamente.

El conjunto de las $12 \mathrm{ANC}$, a pesar de la aparente dispersión, conforma espacialmente dos coronas concéntricas alrededor del centro urbano; una afecta la trama interna de la ciudad; la otra, más externa, sigue prácticamente los límites del tejido urbano municipal. Ambas coronas de renovación deben impulsar la revalorización de la totalidad urbana (figs. 1 y 2 ).

Las ANC que conforman la corona interior están emplazadas en los contornos del Ensanche Cerdà; constituyen lo que se podría calificar de «primer cinturón de revalorización urbana». Su misión es suplir el antiguo tramado urbano y usos desarrollados a finales del siglo xIx: industrias, grandes espacios liberados por infraestructuras ferroviarias y portuarias, viviendas antiguas y de baja calidad; han de actuar de vínculo entre el tejido urbano del ensanche y el de los barrios populares que lo rodean (antiguos municipios absorbidos por el de Barcelona) e inducir su revalorización.

La corona externa se constituye como «cinturón de cohesión urbanística». Los nuevos espacios urbanos se emplazan en los intersticios escasamente ocupados y poco articulados de los barrios periféricos conformados durante las décadas de los años sesenta y setenta. Su misión es consolidar y suturar estos tejidos urbanos, al tiempo que revalorizarlos.

La accesibilidad es característica y condición de su centralidad, por ello el emplazamiento de cada ANC, aparte de estar determinada por las características morfológicas, urbanísticas y sociales de cada zona, está también determinada por las redes de transporte o por las infraestructuras existentes o proyectadas.

Las ANC que configuran la primera corona tienen gran accesibilidad por su buena conexión a la red de transportes públicos urbanos, metropolitanos y regionales. Las de la corona externa son accesibles por su emplazamiento cercano a las grandes arterias de circulación urbana y a los nudos de conexión con las infraestructuras regionales e internacionales como el puerto, el aeropuerto y la red de autopistas (fig. 2)

Las ANC más externas se constituyen como espacios centrales accesibles desde el ámbito metropolitano; las interiores, como los espacios centrales que por su localización han de impulsar la expansión del eje central-comercial de Barcelona. 


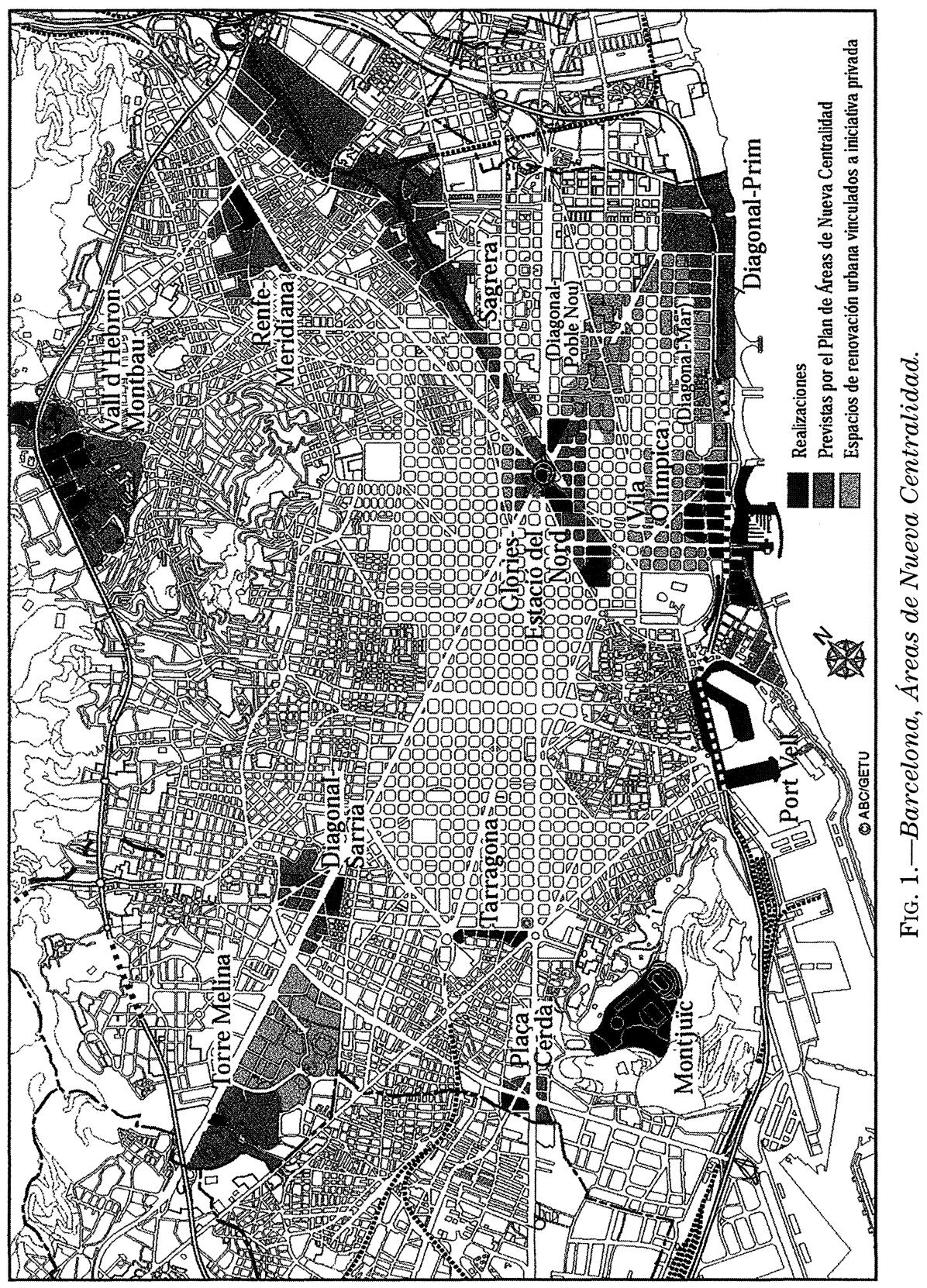




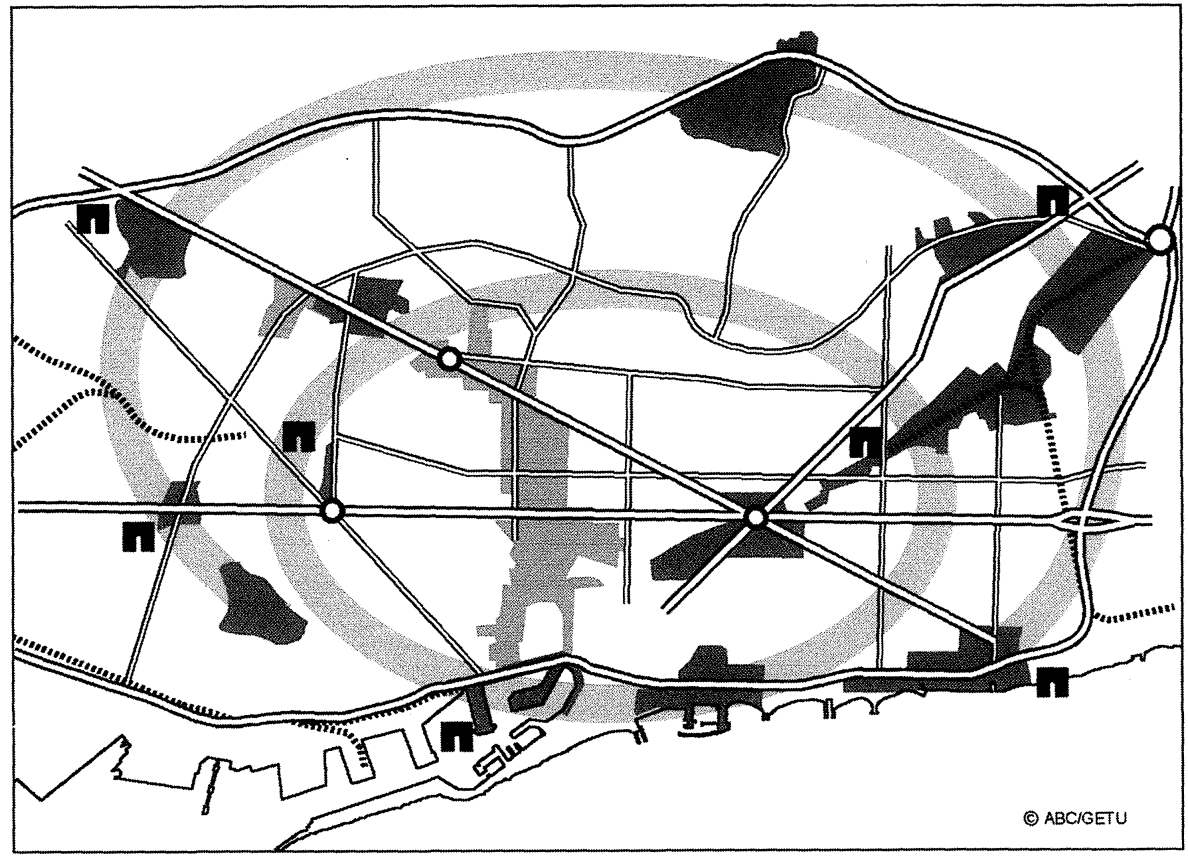

\footnotetext{
* Grandes vías metropolitanas y regionales

* Grandes vías urbanas

" Lineas de ferrocarril

Coronas

1. Portales regionales e internacionales

Eje comercial de los $5 \mathrm{~km}$

Áreas de Nueva Centralidad
}

Fig. 2.-Áreas de Nueva Centralidad. Relación con la red de comunicaciones.

Estrategias de realización. En el Plan de ANC no se define un orden de prioridades para su realización, sólo se establecen los objetivos urbanísticos de cada una y los objetivos generales del Plan, que como se ha señalado, persiguen la transformación del conjunto del espacio barcelonés.

Cada una de las doce ANC está ordenada por un Plan Especial de Reforma Interior (PERI). La flexibilidad de realización y gestión que posi- 
bilita esta figura urbanística a través de las Unidades de Actuación ha permitido a la administración local establecer prioridades de realización en función de la coyuntura política local.

El valor de atracción de los nuevos espacios se origina en su potencial simbólico, basado en el diseño de calidad y en la configuración de elementos morfológicos emblemáticos que las hagan fácilmente identificables. Para ello, el Ayuntamiento ha optado por llevar a término operaciones urbanísticas de prestigio, sirviéndose de los instrumentos urbanísticos que proporcionan los PERI. Desde la oficina de urbanismo se gestiona y controla el proceso de producción de las ANC. Se financia la urbanización del conjunto, incluyendo la construcción de equipamientos y espacios públicos, y se gestiona la totalidad del PERI, desde la negociación con los inversores privados, hasta el control de la calidad estética de los proyectos de urbanización, los estudios de detalle y los proyectos de construcción.

Así pues, cada una de las ANC se caracteriza por la monumentalidad del espacio público y la espectacularidad de la arquitectura como garantías de atractividad para la localización de actividades económicas terciarias de alta productividad; como garantía de la revalorización económica del entorno.

A lo largo de los últimos diez o doce años, se han ido realizando progresivamente diferentes ANC según los requerimientos de las circunstancias político-económicas y según las tensiones de negociación con los agentes económicos y sociales implicados en cada caso.

La celebración de los Juegos Olímpicos fue la circunstancia coyuntural que se aprovechó para impulsar el Plan de ANC. La primeras realizaciones: Villa Olímpica, Área de Montbau, Área de Torre Melina supusieron el primer paso hacia la transformación del espacio barcelonés.

La Villa Olímpica, construida como residencia de los atletas, se ha convertido en una zona residencial de alta calidad y centro terciario con oficinas, actividades comerciales, lúdicas. Emplazada en una antigua zona industrial sus efectos de transformación económica y social ya se han hecho notar: el incremento del precio medio por metro cuadrado de vivienda es uno de los más altos de la ciudad ${ }^{17}$. El Área de Montjuïc, ordenada urbanísticamente para la construcción del anillo olímpico, en la

${ }_{17}$ Tello Robira, R., y Martínez Rigol, S.: «Terciarización y encarecimiento de la vivienda en Barcelona», Revista de Geografía, vol. XXIX, n. ${ }^{\circ} 2$, jul-sept. 1995, pp. 41-51. 
actualidad, es un espacio turístico y de ocio donde tienen lugar espectáculos culturales, lúdicos y deportivos de relevancia. El Área de Montbau reordenada urbanísticamente para ubicar instalaciones deportivas y residenciales relacionadas con los Juegos Olímpicos, aunque no esta finalizada totalmente, hoy ya es zona de equipamientos culturales, deportivos y lúdicos, y, en relación a su emplazamiento cercano al parque natural de Collcerola, se está convirtiendo en un área residencial de alta calidad. El Área de Torre Melina, ordenada para articular el hotel-residencia de autoridades olímpicas con zonas deportivas ya existentes, muy próxima a la zona universitaria, actualmente se ha convertido en un centro de infraestructuras turísticas de alto standing y de grandes equipamientos para el espectáculo deportivo.

Con posterioridad al evento olímpico, el proceso de renovación urbana ha sido mucho más lento, pero sin tregua. Se ha emprendido la realización de ANC vinculadas a actividades de consumo y otras a actividades de negocios.

Las Áreas del Port Vell, Plaça de les Glòries e Illa Diagonal-Sarrià se han desarrollado fundamentalmente como las áreas comerciales que han de contribuir a ampliar el centro comercial de la ciudad, el llamado Eje de los 5 kilómetros o Shopping Line. Cada una se articula a partir de un gran centro comercial que se convierte en el foco de actividad económica que, a su vez, induce la operación de revalorización del entorno. Alrededor del centro comercial se desarrollan diversas actividades lúdicas y culturales que alimentan los flujos de compradores y a la inversa. Complementan estos espacios algunos equipamientos de barrio, tales como escuelas o centros polideportivos, y los espacios públicos peatonales ajardinados que dotan al conjunto del carácter de espacio urbano de calidad o de escenario apropiado para consumir el tiempo libre.

Las ANC calle Tarragona y Plaça Cerdà se concibieron y se están consolidando como dos pequeños CBD, donde son predominantes los edificios para la instalación de oficinas. La primera se construye como eje de negocios, con seis edificios para oficinas, un hotel y un parque público de 4 hectáreas. Este une la estación central de ferrocarriles -Estació de Sants- en conexión con el aeropuerto, el Palacio de Congresos y el recinto de la Feria de Muestras, infraestructuras ya existentes y básicas para el desarrollo de actividades terciarias de gestión.

El Área de la Plaça Cerdà, nudo de arterias locales y regionales, y a la vez, uno de los puntos de conexión entre la ciudad y el aeropuerto, está 
constituida por tres edificios de negocios y espacios públicos ajardinados con equipamientos sociales para los barrios de alrededor.

La única que se ha concebido con una auténtica vocación de equipamiento de barrio es Área de Renfe-Meridiana. Emplazada en la superficie que ocupaban los antiguos talleres de Renfe. Este espacio está destinado fundamentalmente a zona residencial, con equipamientos públicos complementarios: parroquia, escuela, zona deportiva, centro cultural y parque público. No obstante se han previsto tres edificaciones destinadas a usos comerciales y administrativos o de oficinas

A finales de siglo, de nuevo en Barcelona se desarrolla la preparación de otro gran evento: Barcelona 2004: Forum de las Culturas. Otra vez un acontecimiento, que se pretende de resonancia mundial, se utiliza para dar nuevo empuje a la revalorización urbana iniciada para los Juegos Olímpicos. Ahora entran en juego las dos últimas ANC previstas en el Plan de 1987: «Diagonal-Mar» y «Sagrera». Dos áreas que deben inducir a la revalorización socio económica de una de las zonas con menor valor económico de la ciudad.El Área de Diagonal-Mar, emplazada en las proximidades del enlace entre las arterias de circunvalación local y las regionales, con una superficie de 15 ha, se conformará como área de negocios, articulándose con espacios residenciales y equipamientos socio-culturales; asimismo contará con el emplazamiento del nuevo parque zoológico. Con la creación de este nuevo espacio se completa la recuperación de la fachada litoral de la ciudad, iniciada con la construcción de la Vila Olímpica.

El Área de la Sagrera se encuentra pendiente de realización por su vinculación a la estación del tren de alta velocidad. El litigio existente entre el Ayuntamiento, la Generalitat y el Ministerio sobre cual debía ser el trazado del TAV ha retrasado la definición concreta de este espacio, opción que ha defendido el Ayuntamiento por considerarlo vital para la renovación de su entorno. El Área de La Sagrera se concibe también como centro de negocios y espacio lúdico, conformado por una simbología en la que se pretende resaltar la modernidad de las comunicaciones y la tradicionalidad de las estructuras urbanas. Bajo estos criterios, las edificaciones deberán representar la modernidad y el trazado de los espacios públicos deberá respetar y articularse en torno al antiguo trazado de la arteria medieval de suministro de agua a la ciudad, el Rec Comtal. Actualmente se juega con la idea de que el agua constituya uno de los principales elementos simbólicos de los espacios públicos de esta zona (fig. 3). 


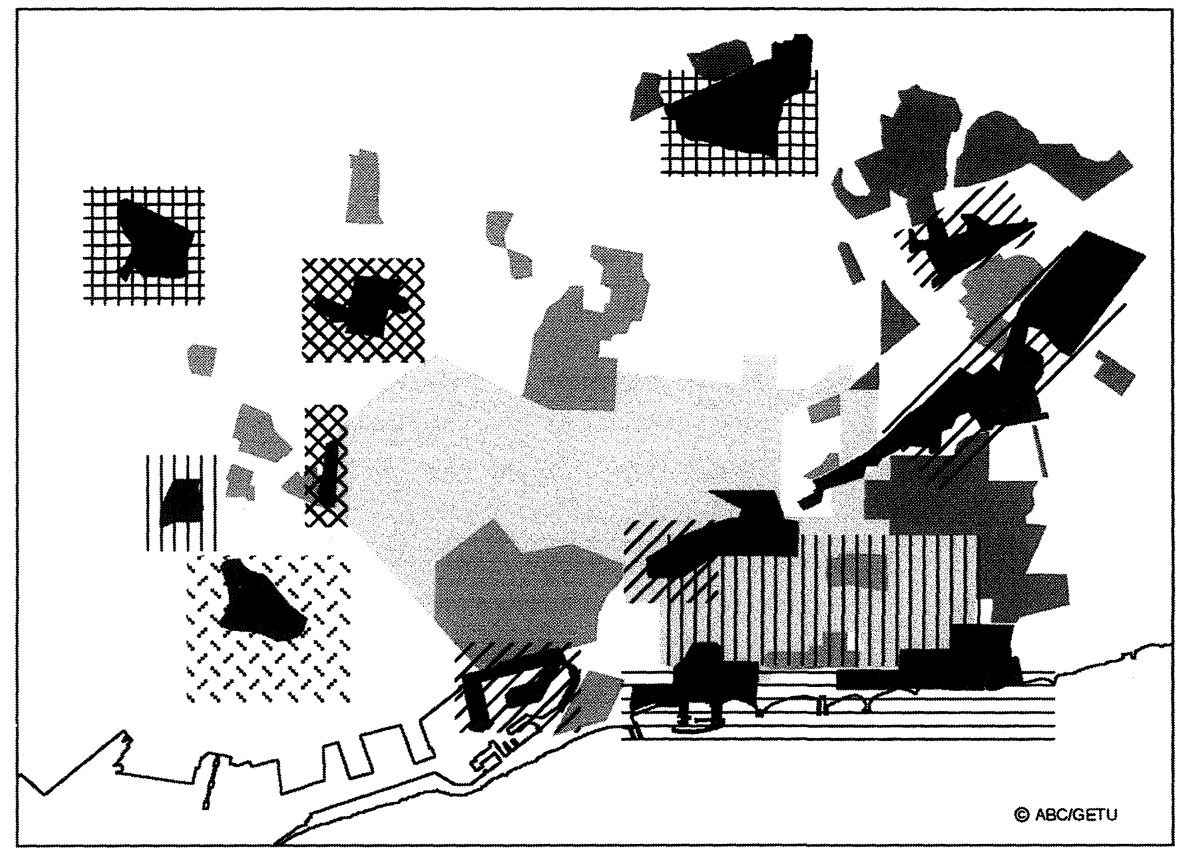

Núcleos urbanos existentes en 1850

Trama urbana correspondiente al Ensanche

Áreas de crecimiento masivo 1950-1975

Áreas de Nueva Centralidad

Renovación y sutura de tejidos urbanos degradados entre barrios

[Z Grandes espacios liberados por infraestructuras obsoletas o en desuso

प1] Tejidos industriales y residenciales obreros degradados

Grandes vacíos urbanos intrersticiales

Recuperación de la franja litoral

Q Recuperación de instalaciones deportivas y espacios libres (Montjuïc)

Fig. 3.-Áreas de Nueva Centralidad y su relación con los tejidos urbanos y usos preexistentes. 
En el sector nordeste de la ciudad es donde se acumulan los mayores «paquetes de actuaciones urbanísticas en torno a las ANC. Aun siendo válidos todos los objetivos definidos hasta el momento, como la descentralización de las actividades terciarias y la dotación de equipamientos, existe un objetivo que no habiendo sido enunciado específicamente, emerge claramente cuando se observa el gran triángulo definido por los vértices Port Vell, Diagonal-Mar y Sagrera: el proceso de gentrification inminente en su interior, es decir un proceso de sustitución social intenso en una de las zonas donde predomina la vivienda, mayoritariamente ocupada por clases populares.

\section{El mercado de oficinas como síntoma de revalorización urbana}

Los efectos multiplicadores que ha tenido la producción de espacios centrales en la transformación de espacio barcelonés se pone en evidencia a través la proliferación de la superficie destinada a oficinas.

El Plan de Áreas de Nueva Centralidad (ANC) confirmó y al mismo tiempo difundió el hecho del déficit estructural de la ciudad en espacio de oficinas: en 1985 había sólo 2,55 millones de metros cuadrados en edificaciones específicamente destinadas a este uso. El mismo Plan se proponía resolver este déficit progresivamente y en la medida que lo iba determinando la demanda. Casi en cada ANC se preveía superficie destinada a oficinas, aunque el uso predominante fuera otro. La iniciativa privada, alertada por el Plan e incentivada por el déficit urbano, dirigió las inversiones inmobiliarias hacia la construcción de oficinas sin contar mucho con las operaciones de remodelación urbana: ya en 1995 se contabilizaban 3,62 millones de metros cuadrados en edificaciones exclusivamente destinadas oficinas, mientras que a mediados de 1999 esta cifra había aumentado a 3,875 millones de metros cuadrados ${ }^{18}$.

Es sintomático como, a partir del año 1988, precisamente poco después de haberse difundido el Plan de Áreas de Nueva Centralidad, crecía de manera espectacular el estoc total de oficinas en la ciudad de Barcelona (fig. 4). Así, atendiendo al número de metros cuadrados construidos dedicados a oficinas, entre 1985 y 1988, el estoc total aumentó en

${ }_{18}$ Larriba, A. (1999): «Falten oficines a Barcelona», en El Periódico de Catalunya: $\mathrm{Ca}$ talunya construeix, 20 de octubre de 1999, pp. 69-70. 


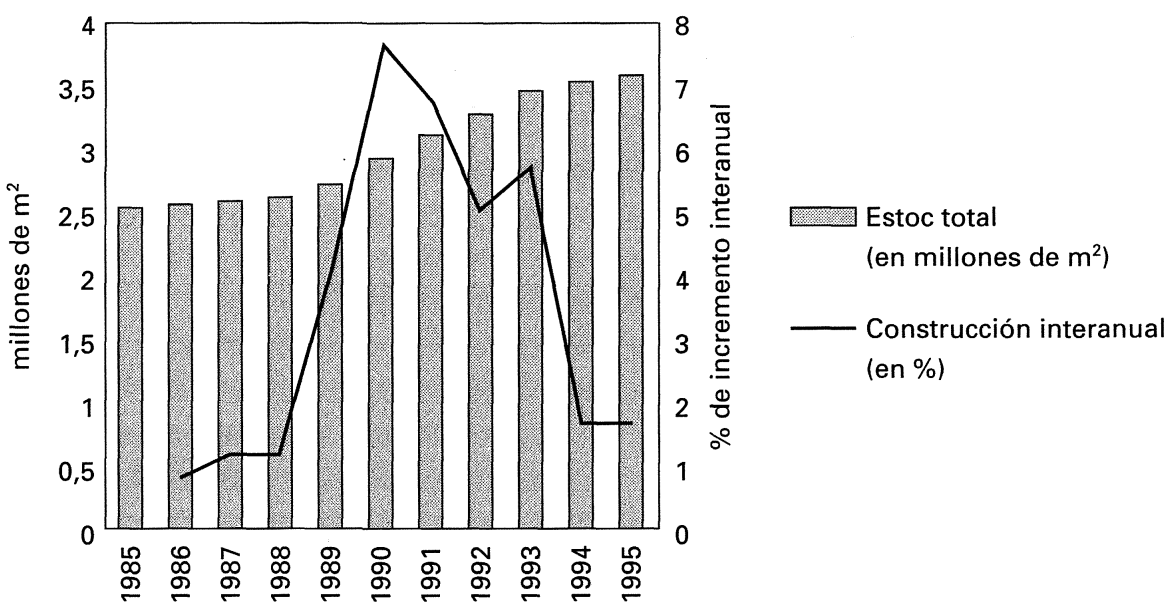

FIG. 4.-Estoc total de superficie dedicada a oficinas y variación interanual, 1985-1995.

unos 80.000 metros cuadrados, mientras que entre los años 1989 y 1993 el incremento fue de 874.000 metros cuadrados, más de un $30 \%$ sobre la que había en 1988. A partir de 1993 la construcción de oficinas seguía creciendo, pero con menor intensidad. En los cinco años posteriores, es decir, entre el 1993 y el 1998, el incremento fue de 300.000 metros cuadrados.

La mayor parte de la inversión tuvo lugar entre los años 1989 y 1993, cuando el sector tenía las máximas expectativas de beneficio, apoyado en el proceso de internacionalización de la ciudad y en un ciclo económico de crecimiento que en España, y concretamente en Barcelona, se invirtió una vez finalizados los eventos de 1992. Así, a partir de 1993, la inversión inmobiliaria se frenó, por un lado debido al período de crisis general que provocaba la retracción de la demanda, pero también debido a la saturación del mercado.

La misma dinámica se confirma si se analiza la cantidad de metros cuadrados de techo dedicado a oficinas previstos en las licencias de obras concedidas por el Ayuntamiento de Barcelona (fig. 5). Es a partir del período 1989-1990 cuando que se dispara el número de metros cuadrados dedicados a oficinas, llegando a representar, en el año 1991, el 17\% del total del techo previsto en la ciudad de Barcelona, porcentaje muy alto teniendo en cuenta que en el año 1989, era sólo el 6\%. Desde 1991-1992 se 


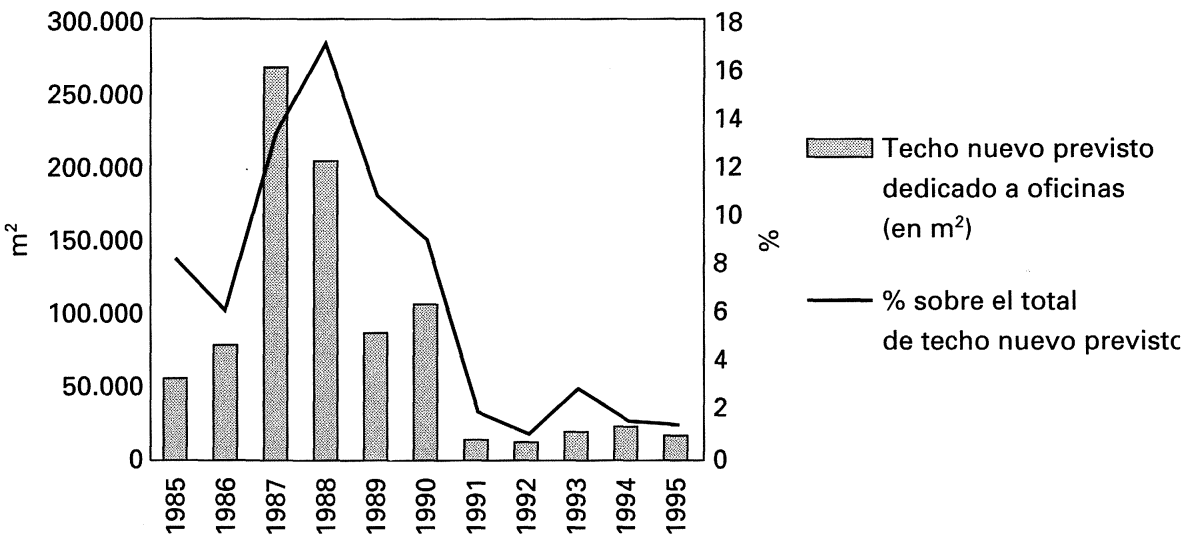

Fig. 5.-Techo nuevo previsto en las licencias de obras dedicado a oficinas, 1988-1998.

inicia una reducción que se radicaliza a partir de 1994, mostrando claramente cómo en los años anteriores ya se había saturado el mercado.

Los promotores inmobiliarios, como cualquier actividad productiva, siguen las inercias inversoras hasta mucho más allá de lo que la racionalidad económica y el planeamiento de los usos urbanos requieren. El resultado es una sobreabundancia de superficie destinada a oficinas que satura el mercado. El síntoma de esta saturación se puede observar a través de la evolución de los precios y la evolución de la desocupación de oficinas.

Entre los años 1991 y 1992, los precios llegaron a su máximo, con una renta mensual de 4.500 pesetas por metro cuadrado. En 1983, esta misma renta se situaba en una media de 750 pesetas por metro cuadrado, mientras que en 1993 cayó a las 2.250 pesetas, precio situado muy por debajo del precio máximo que se había alcanzado al inicio de los años noventa y que se mantuvo con pocas variaciones hasta $19988^{19}$.

A partir del año 1990 comenzó a ser significativa la tasa de desocupación de oficinas. Así, si este año se situaba en torno del 2\%, en 1992 ya estaba en el 6\% y en 1994 era cercano al 14\%. Esta desocupación, como se ha visto, coincidió con los incrementos máximos de los precios y con los momentos de mayor producción de superfície para oficinas. A partir de

${ }_{19}$ Richard Ellis (1999): Global trends. 
1994 la tasa de desocupación empezó a decrecer, hasta situarse en 1999 cercana al $4,75 \%$. Hoy se considera de nuevo que se inicia otra vez una situación de déficit de oficinas, especialmente de las de máxima calidad, lo cual puede volver a generar un ciclo alcista de los precios. Promotores y constructores constatan nuevamente la necesidad de poner en el mercado más oficinas disponibles, aunque alertaban del peligro de saturar otra vez el mercado, como ya sucedió a mediados de los años noventa ${ }^{20}$.

La localización de las promociones inmobiliarias para oficinas es un buen indicador de la revalorización del conjunto del espacio barcelonés. Diversas fuentes, todas ellas parciales, en cuanto al conjunto de las promociones analizadas, y diferentes en cuanto al periodo de tiempo considerado, permiten realizar una aproximación a los efectos espaciales de los nuevos emplazamientos de oficinas. A pesar de la diversidad y la parcialidad de los datos se puede constatar un proceso de descentralización del espacio terciario dedicado a oficinas, tradicionalmente localizado en el eje de $5 \mathrm{Km}$.

Según el informe Boletín informativo sobre el Mercado de Oficinas en Barcelona, elaborado por la compañía Richard Ellis en el segundo trimestre de $1995^{21}$, en el centro de negocios, definido por ellos ${ }^{22}$, se localizaba el 23,39\% (847.000 $\left.\mathrm{m}^{2}\right)$ de la totalidad de la superfície de oficinas; en las ANC del Port Vell, calle Tarragona, Plaça Cerdà, Glòries, Villa Olímpica y Diagonal Mar (esta última aún pendiente de realización en el momento de la publicación del boletín) se localizaba el $3,92 \%\left(142.000 \mathrm{~m}^{2}\right)$, en el resto de la ciudad el $62,28 \%\left(2.276 .000 \mathrm{~m}^{2}\right)$ y el $9,38 \%\left(356.000 \mathrm{~m}^{2}\right)$ restante estaba localizado en la periferia ${ }^{23}$ inmediata de la ciudad de Barcelona. El peso relativo de las ANC en el conjunto de la ciudad era todavía pequeño, mientras que el resto de la ciudad concentraba la mayor parte del espacio dedicado a oficinas, y el centro de negocios mantenía una posición intermedia.

${ }^{20}$ Larriba, A. (1999): «Falten oficines a Barcelona», en El Periódico de Catalunya: Catalunya construeix. 20 de octubre de 1999, pp. 69-70. CAMA, X. (1999): «El nivell del lloguer se situa en un clar cicle alcista», en El Periódico de Catalunya: Catalunya construeix, 20 de octubre de 1999 , p. 71 .

${ }^{21}$ Richard Ellis (1995): Boletín informativo sobre el mercado de oficinas de Barcelona. Segundo trimestre de 1995. Richard Ellis, Barcelona.

${ }_{22}$ El CBD, según Richard Ellis era el área limitada por el passeig de Gràcia hasta la avinguda Diagonal, y en dirección oeste hasta su confluencia con la avinguda de Pedralbes. También incluian el área que envuelve el CBD delimitada por la via Augusta, travessera de Gràcia, Aribau, via Laietana, gran Via, Aragó y las zonas colindantes con la plaça Catalunya. Por lo tanto, se deben considerar las diferencias entre este CBD y el definido a partir del eje comercial de los cinco kilómetros.

${ }^{23}$ Incluía las poblaciones periféricas al municipio de Barcelona como por ejemplo Sant Just Desvern, Sant Joan Despí, Sabadell, Sant Cugat, Terrassa y otras. 
La distribución espacial de la desocupación de oficinas, así como su evolución entre los años 1993 y el 1995, podían hacer pensar que las nuevas áreas de negocios (ANC) se consolidaban como nuevos centros, ya que eran las únicas áreas de toda la ciudad que conseguían disminuir el número total de metros cuadrados desocupados, aunque mantenían la tasa de desocupación más alta de toda la ciudad — probablemente debido a su reciente construcción-, casi del 47\% en el año 1995 (cuadro II). Por otro lado, el «resto de la ciudad» era el área con la menor tasa de desocupación (6,59\%), casi la mitad que la media de la ciudad (11,68\%), aunque los aproximadamente 150.000 metros cuadrados no ocupados representaban, en 1995, más del 35\% del estoc desocupado de toda la ciudad. Por su lado, el distrito de negocios, mantenía una estabilidad en todas las variables: pequeños aumentos del estoc desocupado y de la tasa de desocupación, e imperceptible disminución respecto al total del estoc desocupado de la ciudad.

\section{CUADRO II}

EVOLUCIÓN DE LA TASA DE DESOCUPACIÓN DE OFICINAS ENTRE 1993 Y 1995

\begin{tabular}{|c|c|c|c|c|c|c|}
\hline Área & $\begin{array}{c}\mathrm{M}^{2} \text { deso- } \\
\text { cupados } \\
1993^{*}\end{array}$ & $\begin{array}{c}\mathrm{M}^{2} \text { deso- } \\
\text { cupados } \\
1^{2995} *\end{array}$ & $\begin{array}{c}\text { Tasa de } \\
\text { desocupa- } \\
\text { ción } \\
1993(\%)\end{array}$ & $\begin{array}{c}\text { Tasa de } \\
\text { desocupa- } \\
\text { ción } \\
1995(\%)\end{array}$ & $\begin{array}{c}\% \text { del } \\
\text { total del } \\
\text { estoc deso- } \\
\text { cupado } \\
1993\end{array}$ & $\begin{array}{c}\% \text { del } \\
\text { total del } \\
\text { estoc deso- } \\
\text { cupado } \\
1995\end{array}$ \\
\hline $\begin{array}{c}\text { Distrito de } \\
\text { negocios }\end{array}$ & 89.000 & 92.008 & 10,74 & 10,86 & 22,03 & 21,76 \\
\hline $\begin{array}{r}\text { Nuevas áreas } \\
\text { de negocios }\end{array}$ & 83.000 & 66.550 & 78,30 & 46,87 & 20,54 & 15,74 \\
\hline $\begin{array}{l}\text { Resto de la } \\
\text { ciudad }\end{array}$ & 105.000 & 149.888 & 4,66 & 6,59 & 25,99 & 35,44 \\
\hline Periferia & 127.000 & 114.437 & 39,94 & 32,15 & 31,44 & 27,06 \\
\hline Total & 404.000 & 422.883 & 11,53 & 11,68 & 100 & 100 \\
\hline
\end{tabular}

* Los datos corresponden a «Invierno de 1993» y al «Segundo Cuatrimestre de 1995». Fuente: Boletín informativo sobre el mercado de oficinas de Barcelona, Richard Ellis (1993 y 1995).

El análisis de la localización de las promociones inmobiliarias de nuevas oficinas - a partir del estudio elaborado por el Instituto de Gestión 
de Proyectos ${ }^{24}$-, la mayor parte de ellas resultado de operaciones inmobiliarias de nueva construcción y rehabilitación integral de edificios de alto valor arquitectónico o histórico-patrimonial, permitía constatar la conformación de nuevas áreas de negocios al margen del tradicional eje de los $5 \mathrm{~km}$ (cuadro III). Así, más del 76\% de las nuevas promociones se

CUADRO III

PROMOCIÓN DE EDIFICIOS DE OFICINAS

\begin{tabular}{lrc}
\hline \multicolumn{1}{c}{ Inmueble } & Sup. Oficinas $\mathbf{~ m}^{2}$ & Sup. Comercio $\mathbf{~ m}^{2}$ \\
\hline Barcelona Glòries & 32.000 & 70.000 \\
Edifici Diagonal & 1.588 & \\
Edifici Hammerson & 7.348 & 2.015 \\
Complex Atenea & 3.100 & 3.000 \\
World Trade Centre & 60.000 & 107.000 \\
Diagonal Mar & 220.000 & \\
Torre Mapfre & 45.000 & 2.500 \\
Palau de Mar & 26.000 & 35.000 \\
Illa Diagonal & 48.000 & 1.000 \\
Edifici Omega & 7.200 & \\
Edifici Barcelona 2000 & 10.605 & 2.980 \\
Edifici Roma 25 & 2.586 & 1.000 \\
Palau Malagrida & 2.525 & \\
Edifici Diagonal-Caravel.la & 4.573 & 16.439 \\
Torre Suècia & 13.000 & 17.000 \\
Torres Cerdà & 20.000 & \\
Centre Comercial Maremàgnum & & \\
Edifici El Centre & 2.200 & \\
Edifici Via Augusta & 22.000 & \\
Torre Nuñez i Navarro & 23.400 & \\
Edifici Juan de Austria & 11.341 & \\
Edifici Inverfenix & 1.892 & \\
Caja Madrid & 26.871 & \\
Palau Nou & s.e. & \\
\hline
\end{tabular}

Fuente: Análisis dels operadors econòmics urbans i l'oferta de sostre per a ús terciari, IGP (1995), Barcelona.

${ }^{24}$ IGP (1995): Análisi dels operadors econòmics urbans i l'oferta de sostre per a ús terciari. Barcelona. Policopiado. 


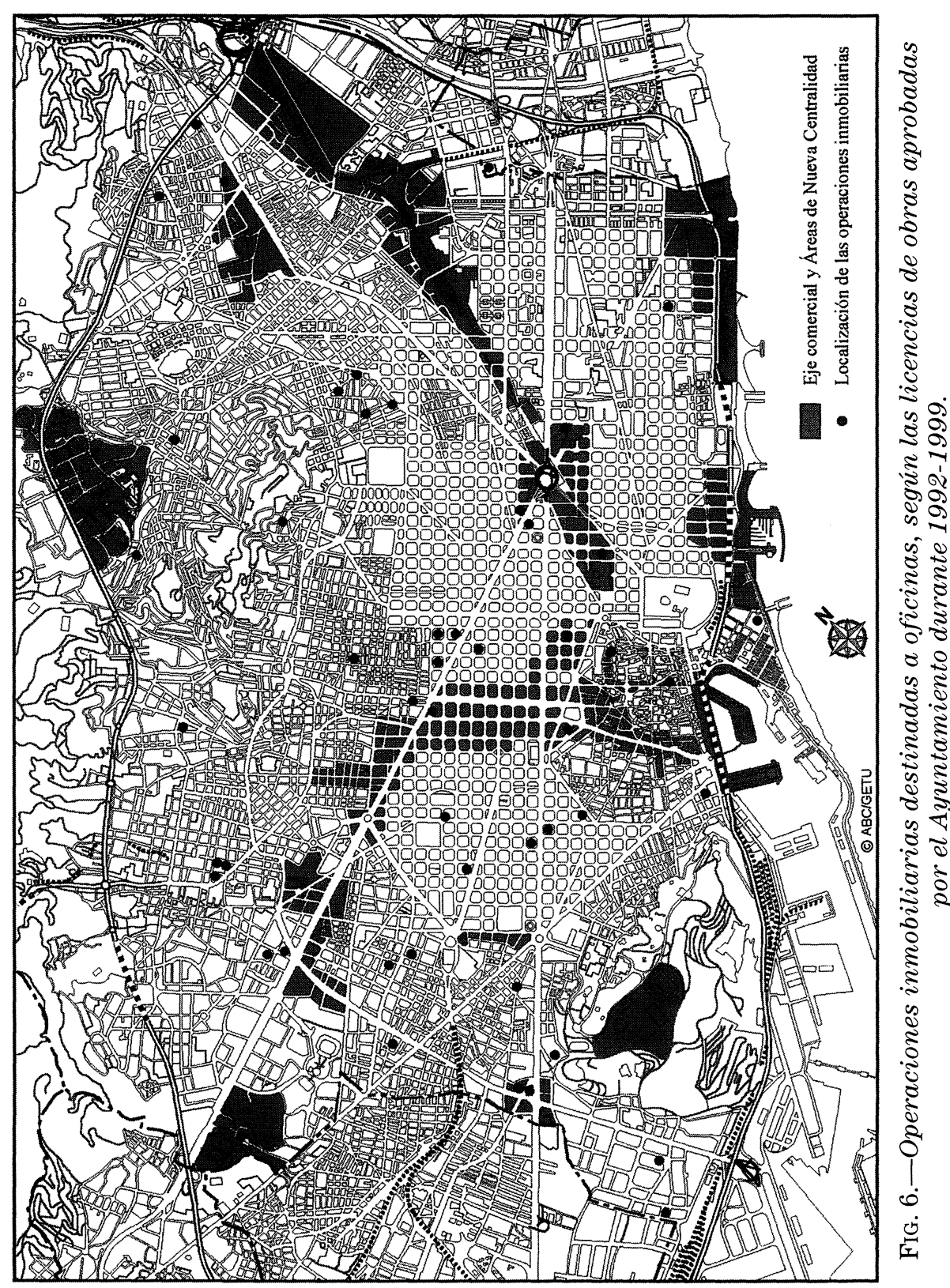


localizan fuera del centro tradicional, mientras que en él sólo se situaban el 24\% de las nuevas promociones. De las primeras, once se localizaban en el interior de alguna de las Áreas de Nueva Centralidad, mientras que las ocho restantes se situaban a su alrededor o bien de forma aislada en otras áreas de la ciudad.

A partir de las promociones de oficinas previstas por las licencias de obras concedidas por el Ayuntamiento de Barcelona, se puede observar como, entre los años 1992 y 1998, la mayor parte de estas promociones no se situaban ni en el tradicional distrito de negocios, ni en las Áreas de Nueva Centralidad (ANC), sino en el resto de la ciudad, algunas claramente alrededor de los dos espacios especializados, el CBD y las ANC (fig. 6). Sólo se aprobaron licencias de obras con techo exclusivamente para oficinas en las Áreas de Nueva Centralidad siguientes: Torre Melina, Glòries, Renfe Meridiana y Plaça Cerdà. En estos años, pues, no se incluyen promociones importantes de oficinas como las del World Trade Center de 20.000 metros cuadrados situadas en el ANC de Port Vell, ni las de Glòries y ni las de la calle Tarragona. Igualmente, en el eje de los $5 \mathrm{~km}$ sólo se dieron cuatro licencias. Las restantes treinta y nueve licencias aprobadas se localizaron en otras áreas de la ciudad, fuera del espacio afectado directamente por las Áreas de Nueva Centralidad o por el eje central comercial y de servicios. Algunas, más o menos estratégicamente localizadas, como en Zona Franca, Sants-avinguda Madrid (cercano a la ANC calle Tarragona), Maria Cristina (cercano a la ANC Diagonal-Sarrià), o Sarrià-via Augusta, pero la mayoría de forma aislada sin aparente relación con el espacio circundante y, a esta escala de análisis, sin aparente estrategia de localización.

La distribución desigual pero indistinta de las operaciones inmobiliarias para uso exclusivo de oficinas dentro del conjunto de la ciudad, las fluctuaciones en la ocupación y en los precios, son indicadores del proceso de transformación de Barcelona en un gran centro de negocios de ámbito metropolitano. Muestra que el impulso que ha realizado en este sentido la administración pública local ha tenido la respuesta de la iniciativa privada. Muestra como la ciudad se ha convertido en una gran área de negocio para el capital inmobiliario, no sólo a través de la construcción de oficinas, sinó también mediante la renovación del parque de viviendas, claro síntoma e la sustitución social que se está produciendo en la ciudad. 
RESUMEN: Se analiza la adaptación del espacio urbano barcelonés al proceso de terciarización. A través de indicadores demográficos, de empleo, precio de la vivienda y rentas familiares se valora la importancia de dicho proceso. Se analizan la ideología y la política urbana que se ha desarrollado en los últimos veinte años, haciendo especial hincapié en las estrategias de producción de los nuevos espacios urbanos denominados Àrees de Nova Centralitat. Finalmente a través del análisis del mercado y localización de la producción inmobiliaria de oficinas se valoran los efectos socio-espaciales del proceso de terciarización.

PALABRAS CLAVE: terciarización; política urbana; reestructuración urbana; centralidad urbana.

RESUMÉ: On analise l'adaptation de l'espace urbain barcelonais au processus de tersiarisation. Des indicateurs sur la démographie, l'emploi, le logémment et les révenues familiales ont permit calibrer l'importance de cet processus. On analise l'idéologie et la politique urbaine qui est à la base des transformations de l'espace urbain de Barcelone pendant les dernieres vingt années. On met l'accent sur les stratégies de production des nouveaux espaces, appellés Àrees de Nova Centralitat. Finalement l'étude de l'inmobilier du bureau et de la localisation des nouvelles constructions ont les a utilisé pour valorer les effets socio-spatiaux du processus de tersiarization.

MOTS CLÉS: tersiarisation; politique urbaine; reestructuration urbaine; centralité des espaces urbains.

ABSTRACT: This paper analyses the restructuring of the Barcelona urban space to the tertiarization process. The importance of this process is assessed through some indicators, such as demographic and occupational ones, household incomes and housing prices. The ideology and the urban politics that have been developed during the last twenty years have also been analysed, stressing the strategies of production of the new urban spaces called Àrees de Nova Centralitat. Lastly, the socio-spatial effects of the tertiary process are assessed through the analysis of the offices market and the location of new office buildings.

KEYWORDS: tertiarization; urban policy; urban restructuring; urban centrality. 